\title{
Reverse cardiac remodeling in patients with dilated cardiomyopathy post Cardiac Resynchronization Therapy (CRT) and its clinical implication
}

\author{
K.E.El Rabat, A.El Naggar, S.I.Farag and A.M.Heikal \\ Cardiovascular, Dept., Faculty of Medicine, Benha Univ., Benha, Egypt \\ E-Mail: hikal.cardio@gmail.com
}

\begin{abstract}
Aim: To track the reverse cardiac remodeling in patients with dilated cardiomyopathy post cardiac resynchronization therapy (ON and OFF modes) and its implication on patients clinical condition emphasizing the factors achieving that remodeling. Patients and Methods: 30 patients indicated for the device were enrolled and studied before and three to six months after device insertion by history taking, clinical examination, transthoracic echocardiogram, ECG and six minutes' walk test. Results: 30 patients (23 males and 7 females, mean age (54.50 \pm 6.24years) with implanted CRT. All patients were in sinus rhythm, QRS duration $\geq 120 \mathrm{~ms}$ and LBBB with dilated cardiomyopathy (ischemic etiology was excluded by coronary angiography in patients having risk factors for IHD). There was clinical response to CRT as NYHA class improved $p<0.001$ and 6 min walk test $p<0.001$, electrocardiographically, there were significant differences in HR, PR interval, QRS duration ,axis and morphology between pre and post CRT measurements $(\mathrm{P}<0.001)$ and echocardiographic response as LV volume improved in $80 \%$, EF in $76.6 \%$ and change ( $\triangle$ QRSD) significantly correlated with changes in NYHA, LVEDD, LVESD, and LVEF. s. Area under the curve was 0.824 for $\triangle \mathrm{QRSD}(\mathrm{p}=0.005), \Delta \mathrm{QRSD}$ of $\geq-20 \mathrm{~ms}$ as a cutoff value had high specificity for predicting both clinical and Echocardiographic response. Coronary sinus lead selection and positioning had a major role in achieving that remodeling with best response was shown with the lateral veins (70\% of responders ) and worst response with mid vein ( $65 \%$ of non- responders);Achieving that targeted significant $\Delta$ QRSD of $\geq-20$ ms sometimes required a more than usual programmable VV delay up to $60 \mathrm{~ms}$ in some patients. Conclusion: Reverse cardiac remodeling post CRT in patients with DCM in both (CRT ON + OFF modes ) was associated with a significant improvement in patients clinical conditions with subsequent reduction of the incidence of their need to hospital admissions.
\end{abstract}

Keywords: Dilated cardiomyopathy, CRT, QRS duration.

\section{Introduction}

The clinical features that were then identified as a HF have been described about 25 years ago. Heart failure is now recognised as a major and evolving public health problem in industrialised countries with ageing populations. One of the most controversial issues about the subject of heart failure at present is the presence of this illness in patients with preserved left ventricular ejection fraction (without any secondary cardiac causes ). There are, however, two recent studies of this type of heart failure. The Olmsted county study, Minnesota, found that $42 \%$ of patients with chronic heart failure had an LVEF $50 \%$. Similarly, the Framingham investigators found that $52 \%$ of their cohort with heart failure had an LVEF of $50 \%$. due to better diagnostics of heart failure as a disease having whole left ventricular contractility spectrum, associations with the growing burden of obesityrelated diseases and with the ageing of the population can be considered. Also, Data tells that the number of patients with heart failure may be on the rise goes up in developing areas under the multiple drawbacks of community diseases and unhealthy lifestyle. As mentioned above, together with that the rate of mortality due to the disease hasn't improved that much , indicate we still have a major problem to deal with[1]. About 64.3 million people are struggling with HF all over the world .19 In developed countries, With a prevalence to be about $1 \%$ to $2 \%$ of the general adult population [2].
Although HF is initially an illness of old populations, last data supported its prevalence in younger populations. Also, the mean age at onset of symptoms declined as well, so the numbers of patients with this syndrome has been doubled from $3 \%$ to $6 \%$, .The exact reason is stiil not known for sure but with the increase in obesity in the current populations with its related catastrophies as DM and HTN with the increasing incidence of atrial arrhythmias as AF [3]. We admit the survival of patients with that syndrome had a great improvement over the last decade with the introduction of recent therapies but those patients still at increased risk of recurrent hospital admissions with the related risks of morbidities and mortalities [3].

Left ventricular ejection fraction (LVEF) is a major tool in classification and defining the type of appropriate treatment. Which has classified the patients into HFrEF and HFpEf . with different estimated and cutoff values for the EF To settle the issue of debate about the modest values between HFrEF and HFpEF the term Mid -range EF was chosen by the cardiology communities [1].

The concept of biventricular pacing is to try to adjust the myocardial dyssynchrony in patients with BBB; so better contractility and stroke volumes Which is believed to improve the symptomatology [4]

Many clinical randomized trials showed that $\mathrm{Bi}$ ventricular pacing has achieved at a six months of follow up almost a $15 \%$ absolute reduction in left ventricular EDD and up to $6 \%$ improvement in LVEF following CRT [4]. 
More than $30 \%$ of patients in whom the device was inserted have shown to be non-re sponders as they failed to show improvement of the NYHA class after 6 ms of follow up[5] QRSD can represent the electrical remodeling og myocardium in HF especially the LV ;So with prolonged QRSD ;Mechanical dyssynchrony is predicted and it can be used to predict the response to the device [6].

2. Aim

To track the reverse cardiac remodeling in patients with DCM post cardiac resynchronization therapy (ON and OFF modes) and its implication on patients' clinical condition emphasizing the factors achieving that remodeling.

\section{Patients and Methods}

thirty patients(30) who were indicated for CRT were enrolled at National Heart Institute(NHI) and Misr University for Science and Technology(MUST) "in the period from October $10 / 2018$ to April (4/2019)". Twenty patients(20) had CRT-Pacemaker devices (Medtronic.); Eight patients had a CRTPacemaker (St Jude Abbott; USA) and two patients had a CRT-D devices (Boston scientific) were studied before and at least three to six months(3-6) after implantation.

Inclusion criteria:

All patients fulfilled all the following criteria:

New York Heart Association (NHYA) functional class III (significant limitation of physical performance . or ambulatory class IV (symptomatic at rest ) Dolgin et al ;March 1994.

With stable hemodynamics for at least one month on max tolerated medications.

Left ventricular ejection fraction (LVEF) less than or equal to $35 \%$ using Echo machine .

Dilated (Non- ischemic) Cardiomyopathy.

Electrical criteria : QRS duration $\geq 120 \mathrm{msec}$ >with LBBB morphology and normal sinus rhythm

The patients were on maximal tolerated and guidelines directed medical therapy

\subsection{Exclusion criteria}

- NYHA class I or II.

- Narrow QRS complex(less than $120 \mathrm{~ms}$ ).

- Ischemic cardiomyopathy (excluded normal coronary angiography).

- Ejection fraction more than $35 \%$.

- Non LBBB morphology.

- Non sinus rhythms (e.g. atrial fibrillation).

Methodology and study procedures:

The assessment included :

\section{History taking:}

Thorough history taking was done with particular stress on the following:

- Symptoms of heart failure

- Symptoms of pulmonary venous congestion e.g. "cough; hemoptysis; orthopnea and paroxysmal nocturnal dyspnea".

- Symptoms of Systemic venous congestion e.g. “ Bilateral lower limbs pitting edema; abdominal distension; dyspepsia)
- Symptoms of low cardiac output (Dizziness; fatigue: claudication pains) etc.

- NYHA functional class: According to the New York Heart Association, the severity of symptoms of Heart Failure can be classified to:

- Class I: No limitation of physical activity; ordinary physical activity does not cause fatigue.

- Class II: Slight limitation of physical activity; comfortable at rest but ordinary physical activity result in fatigue, palpitations.

- Class III: Marked limitation of physical activity; comfortable at rest but less than ordinary activity causes palpitations and fatigue.

- Class IV: Unable to carry on any physical activity without discomfort; symptoms at rest .

- History of hypertension defined in this study by having history of elevated BP more than $140 / 90 \mathrm{mmHg}$ and receiving oral hypertensive agents.

- History of smoking or being a former smoker (meaning that the patient quit smoking more than one year ago).

- History of diabetes mellitus.

- History of frequent hospital admission for decompensated heart failure.

- History of arrhythmias or conduction disturbances.

- History of previous coronary angiography or perfusion scan to diagnose the etiology of heart failure whether ischemic versus dilated cardiomyopathy.

- History of chronic obstructive airway disease or other co morbidities

\subsection{Clinical examination}

Full assessment of the patient general condition and co - morbidities with particular stress on:

1. Blood pressure, heart rate and rhythm.

2. Evidence of sternotomy or infra-clavicular scars (Ischemic etiology is excluded).

3. Basal rales and other signs of pulmonary venous congestion.

4. Additional heart sounds (especially an apical S3 gallop) or murmurs.

5. Lower limb edema, ascites, hepatomegaly and other signs of systemic venous congestion.

3.3. Investigations

ECG acquisition and analysis

Standard 12-lead surface ECGs with a paper speed of $25 \mathrm{~mm} / \mathrm{s}$ and $10 \mathrm{~mm} / \mathrm{mV}$ gain were analyzed:

Prior to implantation.

Three to six months after implantation while setting the device to CRT-off mode.

CRT-on mode was restored after acquiring ECGs. 


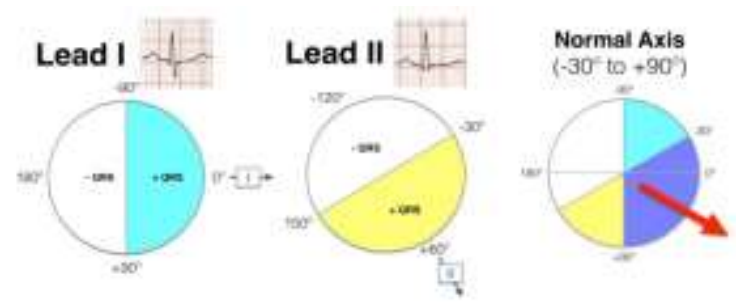

Fig. (1a) QRS axis.

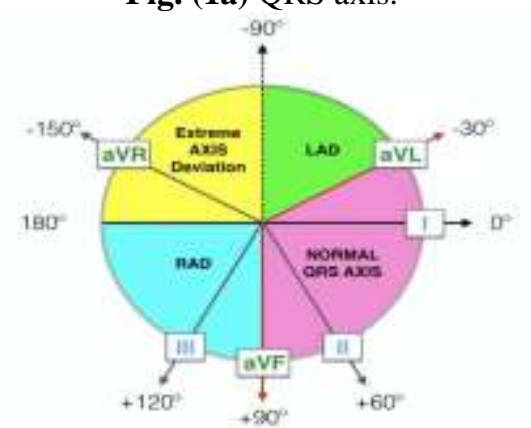

Fig. (1b) QRS axis

\section{The following measurements were recorded :}

1.PR interval (normally 0.12 to $0.20 \mathrm{sec}$ )

2. Intrinsic QRS duration (QRSD) and axis (The entire QRS duration normally lasts from 0.06 to 0.10 seconds).

* Axis There are many different approaches to calculate QRS axis, which are summarized as follow -The Quadrant Method - (Lead I and aVF) The most efficient way to estimate axis is to look at LEAD I and LEAD aVF.

* Three Lead analysis - (Lead I, Lead II and aVF) A positive QRS in Lead I puts the axis in roughly the same direction as lead I.

\subsection{Echocardiography}

using a Vivid 9 Dimension machine with an M4S matrix sector array probe with a frequency range of 1.5 to $3.6 \mathrm{MHz}$ (GE Vingmed Ultrasound, Horten, Norway)

Left ventricular EF, dimensions and volumes (LVEDV \& LVESV) were measured utilizing 2D and $M$ mode .

\subsection{Mitral regurgitation}

MR assessment were assessed before and after CRT implantation according to the latest statements and classified into 1)mild to moderate2)moderate to severe mitral regurgitation according to jet area measurement as follow Mild $<4 \mathrm{~cm} 2$, moderate $4-8 \mathrm{~cm} 2$ and sever $>8 \mathrm{~cm} 2$ ) Left atrium Diameter

Was assessed for every patient pre and at least three months after CRT implantation in the PLAX view.

\section{6. min walk distance}

A reliable objective tool to judge the clinical imorovenment ;in addition to the subjective NYHA class

The test was performed to each patient before and 3-6 months after device implantation.

\subsection{Implantation data}

\section{Fluoroscopic Views:}

- The standard views ( Antero-posterior(AP) ;Left anterior oblique (LAO) and Right anterior oblique( RAO).

- Implantation procedure:

- Sedation and anesthesia : All devices were implanted using local anesthetic"xylocaine" and sedation" Midazolam".

- Venous access: "Using seldinger technique" Left sided approach was applied in all patients ( Most catheters for LV lead placement are designed for the left side

\section{Leads of implantation:}

Right atrial lead:

- Name: (Capsure fix 5076-52; Tendril 52).

- Type of fixation: (All were actively fixated).

- Site: (RA appendage).

- Measurements " average accepted numbers" ( Threshold "1V"; impedance"400 ohm" and amplitude "3v@0.4 msec")

- Right ventricular lead :

- Name: ( Capsure fix 5076-58;Tendril 58;Quick flex 86).

- Type of fixation: ( All were actively fixated).

- Site: ( 26 RV leads were placed in the septal position while $4 \mathrm{RV}$ leads were in the apical position).

- Measurements" average accepted numbers" ( Threshold 1.5v; impedance "500 ohm" and amplitude "3v@0.4 msec").

Left ventricular lead :

Coronary sinus cannulation: ( Direct cannulation of the CS with the introducing catheter ;A diagnostic angiographic catheter ( JR4 or amplatz were used in 3 patients).

CS venography: ( A small amount of contrast "non ionic" was used $30 \mathrm{ml})$.

Projection of views ( LAO;AP and RAO). 
Type of lead :( Attain ability “ 41-42-43” 98-88 LV bipolar ; Attain Performa “42-43-45”98-88 cm LV Quadra ;Optisure and Durata 65).

Coronary vein selection: (the target vessel is the most laterally placed vein in most cases posterolateral, lateral or anterolateral cardiac veins as possible).

Method of implantation: (usually via the subselector only 2 cases where a PTCA wire was used).

Measurements: (Basic settings were performed with testing all the available configuration "Unipolar\ bipolar,LV tip $\backslash R V$ ring”... etc.).

\section{Diaphragmatic stimulation:}

(The output was increased to maximum to check for diaphragmatic stimulation considering that left phrenic nerve stimulation is often positional and may be apparent post-procedure once the patient sat up ; it was accepted to have relatively high thresholds(e.g. $3.5 \mathrm{v}$ at $0.5 \mathrm{~ms}$ ) with $\mathrm{LV}$ pacing if the anatomical site is good

Device programming:

- Battery and leads measurements:( Battery voltage, impedance, longevity and leads impedance).

- Tests:

- RA (sensing and threshold).

- RV (sensing ad threshold).

- LV (threshold).

- Optimization of AV delay:

Altering the AV delay aiming for the following:

Increasing aortic velocity time integral( stroke volume).

Prolongation of diastolic filling: PW Doppler of transmitral blood flow with separation of $\mathrm{E}$ and $\mathrm{A}$ waves.

\section{Optimization of VV delay:}

(In our study we were minded by achieving the VV delay that would achieve the narrowest QRSD) then following the inter and intraventricular dyssynchrony mainly by M-mode septal and posterior wall delay.

\subsection{Complication of CRT}

Complications of CRT implantation include coronary sinus or coronary vein trauma, pneumothorax, diaphragmatic/phrenic nerve pacing, and infection were assessed for all patients

\subsection{Criteria of CRT Response}

After three months post implantation response to CRT was divided into 2 main categories: clinical response and Echocardiographic response.
The clinical response refers to improve clinical status (NYHA functional class by $\geq 1$ NYHA class) and Echocardiographic response refers to decrease in LV end systolic volume by $\geq 15 \%$ and/or increase in ejection fraction by $>10 \%$ [8].

\section{Results}

This Two centers, prospective study enrolled 30 patients (23 males and 7 females, with mean age of $54.50 \pm 6.24$ years years) with CRT device inserted in the period from October 2015 to April 2017. With at least 2 times of assessment 1) at the time of implantation 2) three to six months after device insertion. All patients were in sinus rhythm, with baseline QRS duration $\geq 120 \mathrm{~ms}$ and LBBB morphology and non- ischemic etiology of HF. All programmable data were accepted with $\geq 98 \%$ biventricular pacing.

\section{Demographic data of the studied patients}

-Our research enrolled 30patients 23 males (76.7\%) and 7 females $(23.3 \%)$, with mean age of $54.50 \pm 6.24$. In all patients the underling etiology was non-ischemic.

II. Risk factors: 08 patients $(26.7 \%) \mathrm{Had}$ HTN , 09 patients $(30 \%)$ had DM , 03 patients (10\%) were dyslipidemic and 10 patients (33.3\%) were heavy - smokers.

\section{Clinical response to CRT \\ NYHA class}

15 patient (50\%) improved from NYHA class (III $\rightarrow$ I ), 03 patients (10\%) improved from NYHA class (III $\rightarrow$ II ), 03 patients (10\%) improved from NYHA class (IV $\rightarrow$ I ),02 patients (6.7\%) improved from NYHA class (IV $\rightarrow$ II ) and 02 patients (6.7\%) remained in the same NYHA class and 05 patients deteriorated 3 months after CRT (Table 1).

\section{Six minutes walk test}

- The mean 6MHW distance at baseline was 192.80 \pm 51.65 meters reaching $371.10 \pm 167.20$ meters 3 months after CRT implantation (P value 0.000 ) (Table 2).

- The improvement in the distance was detected in 23 cases who are clinical responders that represent about $78 \%$ of studied cases (Table 2).

Electrocardiographic response to CRT:

All ECG parameters were taken in both modes (with CRT ON + off modes) 3-6 $\mathrm{m}$ after CRT implantation. , there were significant differences in HR, PR interval, QRS duration, axis and morphology between pre and post CRT measurements $(\mathrm{P}<0.001)$.

Table (1) NYHA class improvement 3 to 6 months after CRT.

\begin{tabular}{lll}
\hline NYHA Class Improvement 3 months after CRT & Number \& Percent of Improvement \\
\hline From NYHA class $(\mathrm{III} \rightarrow \mathrm{I})$ & $15(50 \%)$ & \\
From NYHA class $(\mathrm{III} \rightarrow \mathrm{II})$ & $3(10 \%)$ & \\
From NYHA class $(\mathrm{IV} \rightarrow \mathrm{I})$ & $3(10 \%)$ & \\
From NYHA class $(\mathrm{IV} \rightarrow \mathrm{II})$ & & $2(6.7 \%)$ \\
No improvement $(\mathrm{III} \rightarrow \mathrm{III})$ & $2(6.7 \%)$ & \\
Deterioration (III $\rightarrow \mathrm{IV})$ & & $5(16.7 \%)$ \\
\hline
\end{tabular}


Table (2) Difference between pre and post CRT regarding six minute walk distance.

\begin{tabular}{|c|c|c|c|c|}
\hline & Six Minute Walk Distance & & & Paired t-test \\
\hline & Range & Mean \pm SD & $\mathbf{t}$ & p-value \\
\hline Before & $100.00-200.00$ & $192.80 \pm 51.65$ & & \\
\hline After & $190.00-450.00$ & $371.10 \pm 167.20$ & -5.953 & 0.000 \\
\hline
\end{tabular}

Table (3) Electrocardiographic response to CRT(on- mode) in all patients after implantation.

\begin{tabular}{llc}
\hline AFTER IMPLANTATION & No.=30 \\
\hline HR & Mean \pm SD & $89.70 \pm 11.73$ \\
PR & Range & $75-120$ \\
& Mean \pm SD & $123.17 \pm 17.14$ \\
& Range & $70-150$ \\
QRS morphology & QBBB & $2(6.7 \%)$ \\
& RBBB( pure Lv acing) & $2(6.7 \%)$ \\
QRS Width & RS (biventricular) & $3(10.0 \%)$ \\
& Mean \pm SD & $23(76.7 \%)$ \\
Axis & Range & $135.00 \pm 16.66$ \\
LV percentage (\%) & Left & $90-170$ \\
& Right & $27(90.0 \%)$ \\
AV & Range & $3(10.0 \%)$ \\
& Mean \pm SD & $99.53 \pm 0.68$ \\
VV & Range & $98-100$ \\
& Mean \pm SD & $126.50 \pm 6.58$ \\
Axis of LV pacing & LV TIP to CAN & $110-130$ \\
& Tip to ring & $27.00 \pm 11.49$ \\
& & $20-60$ \\
& &
\end{tabular}

Table (4) QRSW with CRT ON and OFF modes.

\begin{tabular}{|c|c|c|c|c|c|}
\hline ECG & QRS WIDTH (On) & QRS WIDTH (OFF) & Test value & P-value & Sig \\
\hline $\begin{array}{l}\text { Mean } \pm \text { SD } \\
\text { Range }\end{array}$ & $\begin{array}{c}122.50 \pm 23.06 \\
90-175\end{array}$ & $\begin{array}{c}129.80 \pm 19.66 \\
100-175\end{array}$ & 4.656 & 0.000 & HS \\
\hline
\end{tabular}

Table (5) Electrical remodeling 3-6 months post CRT implantation.

\begin{tabular}{|c|c|c|c|c|c|c|}
\hline ECG & & Before implantation & 3-6 Months & Test value• & P-value & Sig \\
\hline HR & $\begin{array}{l}\text { Mean } \pm \text { SD } \\
\text { Range }\end{array}$ & $\begin{array}{l}94.53 \pm 10.47 \\
70-107\end{array}$ & $\begin{array}{l}83.17 \pm 8.77 \\
70-100\end{array}$ & 5.273 & 0.000 & HS \\
\hline PR INTERVAL & $\begin{array}{l}\text { Mean } \pm \text { SD } \\
\text { Range }\end{array}$ & $\begin{array}{l}140.00 \pm 6.29 \\
100-200\end{array}$ & $\begin{array}{l}215.00 \pm 16.16 \\
120-260\end{array}$ & 10.019 & 0.000 & HS \\
\hline QRS WIDTH (On) & $\begin{array}{l}\text { Mean } \pm \text { SD } \\
\text { Range }\end{array}$ & $\begin{array}{l}146.83 \pm 15.84 \\
125-180\end{array}$ & $\begin{array}{l}122.50 \pm 23.06 \\
90-175\end{array}$ & 5.193 & 0.000 & HS \\
\hline QRS WIDTH ( OFF) & $\begin{array}{l}\text { Mean } \pm \text { SD } \\
\text { Range }\end{array}$ & $\begin{array}{l}146.83 \pm 15.84 \\
125-180\end{array}$ & $\begin{array}{l}129.80 \pm 19.66 \\
100-175\end{array}$ & 4.455 & 0.000 & HS \\
\hline
\end{tabular}




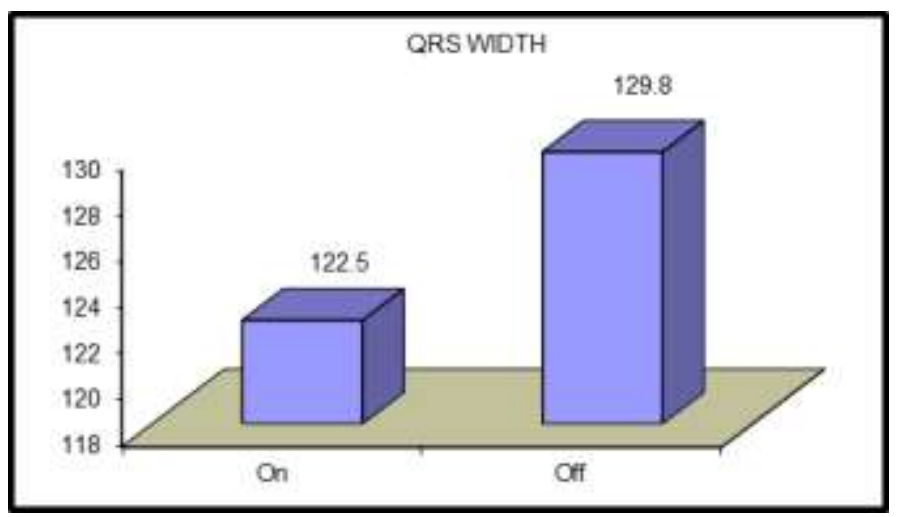

Fig. (2) QRS 3-6 m after CRT with both ON-and OFF.

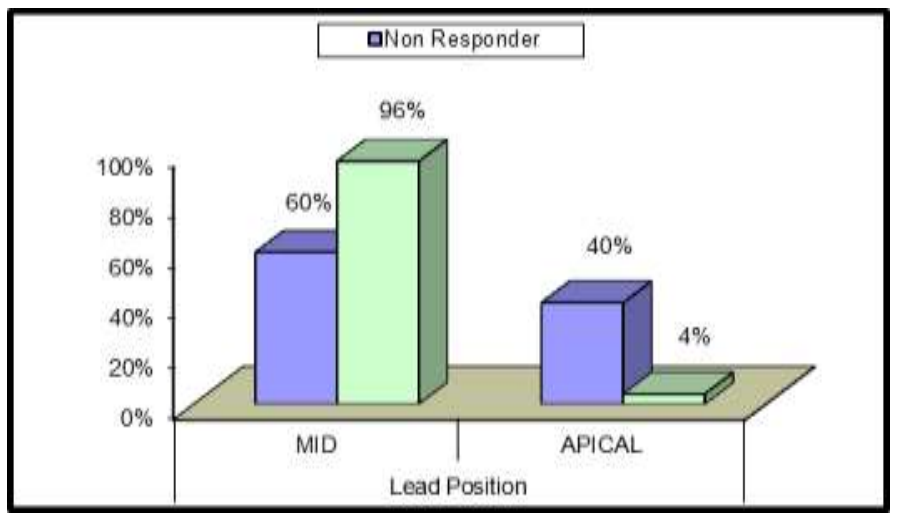

Fig. (3) Position of CS vein in responders and non-responders.

\section{Position of CS vein:}

Basal to mid coronary sinus vein lead positioning showed better response results as shown in Fig (3)

\section{Echocardiographic response to CRT}

a. The mean LVEF at baseline was $26.77 \pm$ $6.13 \%$ reaching $35.43 \pm 9.08,3$ to six months following the device insertion ( $p$ value 0.000 ).

b. The mean LVEDD at baseline was $8.97 \pm$ $10.99 \mathrm{~cm}$ reaching8.12 $\pm 11.16 \mathrm{~cm}, 3$ to six months months following the device insertion ( $p$ value 0.000$)$.

c. The mean LVESD at baseline was $7.79 \pm 9.90 \mathrm{~cm}$ reaching $6.93 \pm 10.07 \mathrm{~cm}, 3$ to six months following the device insertion ( $p$ value 0.000 ).

d. The mean LVEDV at baseline was $280.20 \pm$ $70.27 \mathrm{ml}$ reaching $247.50 \pm 72.98 \mathrm{ml}, 3$ months after CRT implantation (p value 0.000 ). e. e. The mean LVESV at baseline was $181.30 \pm$ $41.08 \mathrm{ml}$ reaching $129.30 \pm 44.11 \mathrm{ml}, 3$ months after CRT implantation ( $\mathrm{p}$ value 0.000 ).

f. The change in MR severity was statistically nonsignificant ( $P$ value 0.076)

g. The mean $\mathrm{Lt}$ atrium at baseline was $5.74 \pm$ $7.43 \mathrm{~cm}$ reaching $5.61 \pm 7.27 \mathrm{~cm}, 3$ months after CRT implantation (p value 0.003 ).

h. As regard the LV EF; patients with marked improvement with $10 \%$ or more increase in $\mathrm{EF}$ were 23 representing $76.6 \%$ of case study

i. Left ventricular stroke volumes showed a siginificant values after implantation $\mathrm{P}$ value was 0.002 [Table 6]

Table (6) echocardiographic response after CRT.

\begin{tabular}{|c|c|c|c|c|c|c|}
\hline Echo & & Before implantation & 3-6 months & Test value & P-value & Sig \\
\hline LA & $\begin{array}{l}\text { Mean } \pm \text { SD } \\
\text { Range }\end{array}$ & $\begin{array}{l}5.74 \pm 7.43 \\
3.6-45\end{array}$ & $\begin{array}{l}5.61 \pm 7.27 \\
3.5-44\end{array}$ & $3.303 \bullet$ & 0.003 & HS \\
\hline EDD & $\begin{array}{l}\text { Mean } \pm S D \\
\text { Range }\end{array}$ & $\begin{array}{l}8.97 \pm 10.99 \\
6-66\end{array}$ & $\begin{array}{l}8.12 \pm 11.16 \\
4.5-67\end{array}$ & $6.350^{\bullet}$ & 0.000 & HS \\
\hline $\mathrm{EDV}(\mathrm{ml})$ & $\begin{array}{l}\text { Mean } \pm \text { SD } \\
\text { Range }\end{array}$ & $\begin{array}{l}280.20 \pm 70.27 \\
180-415\end{array}$ & $\begin{array}{l}247.50 \pm 72.98 \\
156-390\end{array}$ & $4.555^{\bullet}$ & 0.000 & $\mathrm{HS}$ \\
\hline
\end{tabular}




\begin{tabular}{|c|c|c|c|c|c|c|}
\hline \multirow{2}{*}{$\mathrm{ESD}(\mathrm{mm})$} & Mean \pm SD & $7.79 \pm 9.90$ & $6.93 \pm 10.07$ & \multirow{2}{*}{$6.181 \bullet$} & \multirow{2}{*}{0.000} & \multirow{2}{*}{ HS } \\
\hline & Range & $4.9-60$ & $3.5-60$ & & & \\
\hline \multirow{2}{*}{$\mathrm{ESV}(\mathrm{ml})$} & Mean \pm SD & $181.30 \pm 41.08$ & $129.30 \pm 44.11$ & \multirow{2}{*}{$6.800^{\bullet}$} & \multirow{2}{*}{0.000} & \multirow{2}{*}{$\mathrm{HS}$} \\
\hline & Range & $118-264$ & $52-230$ & & & \\
\hline \multirow{2}{*}{ FS } & Mean \pm SD & $12.93 \pm 3.27$ & $16.77 \pm 3.87$ & \multirow{2}{*}{$-6.359 \bullet$} & \multirow{2}{*}{0.000} & \multirow{2}{*}{$\mathrm{HS}$} \\
\hline & Range & $5-17$ & $10-24$ & & & \\
\hline \multirow[t]{2}{*}{$\mathrm{EF}$} & Mean \pm SD & $26.77 \pm 6.13$ & $35.43 \pm 9.08$ & \multirow[t]{2}{*}{$-6.966 \bullet$} & \multirow[t]{2}{*}{0.000} & \multirow[t]{2}{*}{ HS } \\
\hline & Range & $\begin{array}{l}12-35 \\
3(100 \%)\end{array}$ & $\begin{array}{l}19-47 \\
7(233 \%)\end{array}$ & & & \\
\hline \multirow{3}{*}{ MR Grade } & Mild & $6(20.0 \%)$ & $12(40.0 \%)$ & \multirow{3}{*}{$6.850 *$} & \multirow{3}{*}{0.076} & \multirow{3}{*}{ NS } \\
\hline & Moderate & $11(36.7 \%)$ & $5(16.7 \%)$ & & & \\
\hline & Severe & $10(33.3 \%)$ & $6(20.0 \%)$ & & & \\
\hline
\end{tabular}

•: Paired-t test; *: Chi-square test

HS: Highly significant; S: Significant; NS: Non significant

\section{Complications related to CRT implantation}

Three Patients $(0.9 \%)$ had immediate complications after device insertion as the following : 1 patients had hematomas at site of implantation representing $(0.3 \%), 2$ had diaphragmatic pacing which represented $(0.6 \%)$

\section{Discussion}

Cardiac resynchronization therapy (CRT) reduces morbimortality and improves heart failure symptoms in appropriately selected candidates [6].

There are some features that could predict the likelihood of nonresponse to CRT as in patients with ischemic cardiomyopathy (ICM), electrical abnormality other than the typical left bundle branch block, , QRSW less than $120 \mathrm{~ms}$, and delayed activation at the site of left ventricular (LV) lead implant

Despite improvements in guiding LV lead implantation using both electrical and imaging strategies, About $30 \%$ of patients still shows a non response to the biventricular pacing. So , It needs more studying to understand the patterns of electrical remodeling in those who are not responders[26]

The idea of CRT is to restore the electromechanical ventricular synchrony. Initially, the baseline electrical morphology the patient is the primary indicator to response, but also the effect of biventricular pacing is also plays a vital role in this aspect. This later effect is poorly studied [27]

The aim of our work was To track the reverse cardiac remodeling in patients with dilated cardiomyopathy post cardiac resynchronization therapy( ON and OFF modes ) and its implication on patients clinical condition emphasizing the factors achieving that remodeling.

In our study there were significant changes in the electrical patterns on surface ECG after $\mathrm{Bi}-\mathrm{V}$ pacing. Those changes were also evident between the two groups especially the QRSD, morphology, and Axis with different programmable parameters as

(AV and VV delay). This went parallel to the improvement of the symptomatology and the mechanical features
Regarding CRT response:

According to our study, 3 patients had clinical improvement without criteria of echocardiographic response and what worth mentioning that those 3 patients had a narrower QRS complex and less LV dyssynchrony than those who showed both clinical and mechanical respose. Similar observation was found by Dominique Auger ;et al in the study that included a 440 consecutive heart failure patients (mean age $66 \pm$ 11 years, $81 \%$ men) treated with $\mathrm{Bi}-\mathrm{V}$ pacing followed for 6 months, improvement of symptoms was observed in $84 \%(n=370)$ of the patients. Significant lowering in LVESV was noted in 63\% $(n=276)$. Many patients who improved clinically did show LV reverse remodeling $(72 \%, \mathrm{n}=268)$. But , $28 \%(\mathrm{n}=$ 102 ) of patients who improved clinically did not show that parameter [28].

Patients who were considered responders in this study had $\geq 15 \%$ reduction in LVESV which is considered the hallmark of $\mathrm{LV}$ reverse remodeling. This agrees with the study of J. van 't Sant who stated in his study which included a 205 CRT patients (age $65 \pm 12$ years, $69 \%$ men $)$ that $\triangle \operatorname{LVESV}(\geq 15 \%$ reduction in LVESV was considered as the cutoff point) was the most reliable indicator to tell the response to bi-ventricular pacing. Especially in the non-ischaemic aetiologies, this parameter showed to be an important clue to judge the long termed assessment [29]_

The main findings in our study as regard the electrical remodeling post CRT in patients whose baseline surface ECG is LBBB ( as an inclusion criterion) are the intrinsic i-PR interval ; IQRSD; QRS morphology, eventually QRS axis and at last HR. CRT:

Regarding PR interval and QRS duration post

Although prolongation in the PR interval is considered as a benign issue ; Many studies correlate between its presence and the presence of poor prognosis in heart failure regardless the device implantation .

It has a major effect on patients hemodynamics especially in heart failure patients. A short PR interval causes separation of mitral A waves. While, prolongation of the PR interval leads to the fusion of mitral E- and A-waves with diastolic MR . Both affect the LV diastolic filling with reduction of the COP. 
Recently, several automated algorithms designed to adjust the AV or inter-ventricular (VV) delay, such as Sync AV (Abbott, Sylmar, CA, USA), SonR (Sorin Group, Saluggia, Italy), and the Adaptiv CRT algorithm (Medtronic Inc., Minneapolis, MN, USA) have been advocated to improve the response to $\mathrm{Bi}-\mathrm{V}$ pacing. But, to achieve those algorithms, the intrinsic -PR interval needs to be within that relatively short range $(<220 \sim 250 \mathrm{~ms})$. As in patients with prolonged PR intervals, those algorithms could not be used. So ; we hypothesized that shortening the intrinsic PR interval through the $\mathrm{Bi}-\mathrm{V}$ pacing, could allow those algorithms to be used on more patients.

Surprisingly the changes in the intrinsic PR intervals couldn't be correlated with the device response . But even, the i-PRi was observed to increase further and in the responder group as well ;it worth mentioning that the increase was more evident in the non-responder group(with notification that all patients had almost similar doses of beta blockers and only 2 patients were described amiodarone)..

QRSD measured immediately post CRT implantation and 3-6 months post implantation with both CRT -ON and CRT -OFF “ intrinsic QRSD “ modes decreased significantly in the CRTresponder group.

Many previous studies showed that CRT induced both electrical and structural reverse remodeling, where narrowing of the $\mathrm{i}-\mathrm{QRSd}$ was the main indicator of remodeling. The responders showed that changes at the two levels of their i-QRSd during the follow-up, And the acute reduction in $\mathrm{p}-\mathrm{QRSd}$ achieved by $\mathrm{Bi}-\mathrm{V}$ pacing:

In a prospective study of 85 CRT patients, Sebag et al. showed that shortening of the i-QRSd >_ $20 \mathrm{~ms}$ was associated with better symptomatology and mechanical improvement after 12 months of close follow up of patients[26].

Hee-Jin et al also reported in his CRT registry which enrolled 100 patients with NSR and more than 12 months of follow up, intrinsic -QRSW lowered significantly $\left(170 \_39\right.$ to $\left.159 \_24 \mathrm{~ms}, \mathrm{p}=0.012\right)$. but , the intrinsic PR interval was not decreased in whom achieved a CRT response and even increased initially , The super-responder group ( $\mathrm{n}=33)$ Moreover, lengthening of the intrinsic PR interval was observed regardless the CRT response situation or use of medications . But CRT non-responders showed a remarkable PR prolongation $(\mathrm{p}=0.005)$ and $\mathrm{QRSW}(\mathrm{p}=$ $0.001)$, together with positive myocardial remodeling[27]

On the other hand, The effect of prolonged PR interval wasn't studied aggressively or adequately to show its implication on symptomatology ;apart from the (CARE-HF) trial: a longer PRi observed at baseline and at 3 months post-CRT predicted all-cause mortality and HF-hospitalization during follow-up. Further studies are needed to track that effect[28]

On the contrary; Stockburger, et al. did not record any decrease in unpaced QRSW after $\mathrm{Bi}-\mathrm{V}$ pacing in
42 patients $(64+/-9$ years with left ventricular ejection fraction $25+/-8 \%, 16$ coronary artery disease, 26 non-ICM , 21 with LBBB and CRT indication vs 21 controls [matched for gender, age, LVEF, and underlying disease]) an non -paced electrocardiogram and echocardiogram were recorded at baseline (bl) and after $20.6+/$ - 13.8 months (fup) it may also increased( QRS increased in both groups (CRT bl $165+/-22$ vs fup $171+/-20 \mathrm{~ms}, \mathrm{P}=0.07$, controls bi $111+/-17$ while $118+/-19 \mathrm{~ms}, \mathrm{P}=0.01$ ). That conflict could be due to : a) Almost $30 \%$ of the patients were on amiodarone which affects IV conduction, b) Usage of different ways of electrocardiography interval recording $\mathrm{t}$ (Stockburger et al relied on computed measurement, while our study relied on manual method), c) Heterogeneous time period of heart failure before implantation; longer duration might be associated with irreversible damage[29]

However, The response to the device was strongly associated with the remodeling as regards the intrinsic QRSW ( Table 19,diagram 27 ). SO as mentioned above,We can assume that those effects on electrical remodeling could be best achieved at the interventricular level rather than the atrio-ventricular one.

The exact explanation for why intrinsic PRi and QRSw show different responses to the device still not that clear, Here some possibilities :

1. the QRSw resembles the total duration of ventricular activation and is related to the left ventricular dimensions in patients with cardiomyopathy with LBBB. So, lowering of the intrinsic QRSw could be caused partially by structural reverse remodeling of the ventricular myocytes, with the observation of a high correlation between percent reductions in the two parameters .

2. Different anatomic variation in the ANS between the supraventricular and ventricular components is another important factor.. There is an autonomic imbalance, as, sympathetic over activity and parasympathetic withdrawal, can be alleviated or reversed modulated in responders to the device . Beinig more innervated the supraventricular components are more affected by the restored parasympathetic tone post device insertion which could explain that difference. (DeMazumder .et al.,2015)

And at last QRSW doesn't get changed with the variability of heart rate ; Unlike the intrinsic PR intervals. [ 30].

As regard QRS morphology and Axis:

In our study patients who achieved a persistent biventricular pacing pattern (Rs morphology) was associated with both clinical and mechanical" echocardiographic "improving; It is important to highlight that this finding goes in parallel with the Reduction of the QRSD either the paced or the intrinsic (i.QRSD) e.g. Patients who failed to achieve this QRS 
morphology showed a non improved i- QRSD and ended eventually to be a CRT non-responders.

\section{As regard The QRS Axis}

We believe that an important correlation between the lead position and the pre-implantation QRS ellectrical axis's, such that there was a better response to resynchronization therapy when the lead was implanted in the Anterolateral ; postero-lateral ;lateral veins ; Anterior inter-ventricular vein if the patient had a left QRS axis deviation with better results at the Mid rather than the apical lead position .

therapy when the lead was implanted in the Anterolateral ; postero-lateral ;lateral veins ;Anterior inter-ventricular vein if the patient had a left QRS axis deviation with better results at the Mid rather than the apical lead position .

Our results go in agreement with the results of the prospective, observational study done by Javier et al which involved 80 patients; Where the patients had a functional class with NYHA III to IV with reduced left ventricular EF <35\%) and a QRS duration of $>120 \mathrm{~ms}$. The results gives preference to the anterior or lateral veins in patients with a left-deviated QRS axis. Also, with respect to location in the posterior vein, better results were seen in patients with a narrow $\mathrm{QRS}$ axis $(\mathrm{P}=.026)$. Assumed to be due to the different pattern of ventricular activation that In relation to the native complex. Also it was noticed that more delay in activation occured in the upper and lateral positions of the LV in patients with a left-deviatted QRS [7]

As regard $\mathrm{HR}$ :

There were no major changes in HR on the ECG after Bi-V pacing. Which goes with the results of Henrikson and Sebag who reported similar results. Medical treatment for all patients with chronotropic effect may explain this finding and most of them had the same averages of AV delay

In our study 18 patient $(60 \%)$ improved from NYHA class (III $\rightarrow$ I), 3 patients $(10 \%)$ improved from NYHA class (III $\rightarrow$ II), 3 patients $(10 \%)$ improved from NYHA class (IV $\rightarrow$ I ), 2 patients (7\%) improved from NYHA class $(\mathrm{IV} \rightarrow \mathrm{II})$ and 7 patients $(23.7 \%)$ showed a no improvement or even deterioration in the NYHA class 3 months after CRT.

\section{References}

[1] Amy Groenewegen, Frans H.Rutten .Epidemiology of heart failure European Journal of Heart Failure .vol. 22, pp.1289-1492,2020.

[2] E S.Evelien van Riet, Arno W.Hoes, Kim P Wagenaar. The prevalence of heart failure and ventricular dysfunction in older adults over time Eur J Heart Fail.vol. 18(3),pp.242-52,2016.

[3] A.Mostard, AW.Hoes. Clinical epidemiology of heart failure. Heart.vol. 93(9),pp.1137-1146. ,2007.
Those results goes with Ibrahim et al where 192 patients were subjected to $\mathrm{Bi}-\mathrm{v}$ pacing (Guidelines directed insertion). Pre and 3-6 months post insertion clinical and mechanical .Showing a signifiicant improvement of NYHA class after the device (P value $<0.001), 72.2 \%$ of patients improved with at least 1 NYHA class, $61.6 \%$ improved in one NYHA class, $10.55 \%$ showed an improvement of two NYHA classes and $27.8 \%$ did not improve in NYHA class after CRT implantation

In our study, the mean 6MHW distance at baseline was $192.80 \pm 51.65$ meters reaching $371.10 \pm 167.20$ meters 3 months after CRT implantation ( $P$ value 0.000 )

The improvement in the distance was detected in 23 cases who are clinical responders that represent about $78 \%$ of studied cases

. The study of (Yu et al., 2013, Chau et al.,2002) showed a similarity with ours as they studied 25 patients with NYHA class III to IV HF and QRS complex duration>140 ms with bi-ventricular pacing therapy and assessed three months after pacing, mechanically ,six minutes walk test were performed. With improvement in left ventricular (LV) enddiastolic $(205+/-68$ versus $168+/-67 \mathrm{~mL}, \mathrm{P}<0.01)$ and end-systolic volume $(162+/-54$ versus $122+/-42 \mathrm{~mL}$, $\mathrm{P}<0.01)$; and improved 6-minute walk distance after pacing for 3 months which was ana important implicator for a good clinical response[30]

\section{Conclusion}

CRT induces reverse electric remodeling manifested in the surface ECG. CRT responders showed more reduction in native QRS duration than non responders. Reverse electric remodeling was associated with reverse structural remodeling by Echocardiography and Clinical improvement. The selection criteria plays a major role in determining the response to CRT with preference to female gender; the more wide the QRS complex pre implantation the higher probability to be a CRT responder .We recommend that the operator should target the electrical remodeling mainly targeting the narrowest QRS width through selection of the proper coronary sinus vein and adjusting the programming data e.g. ( VV delay).

[4] Mj.Ackerman, SG.Priori, S.Willems, C.Berul, R.Brugada. HRSIEHRA expert consensus statement on the state of genetic testing for the chanalopathies and cardiomyopathies: this document was developed as partnerships between the Heart Rhythm Society (HRS) and the European Heart Rhythm Association (EHRA). Heart Rhythm.vol. 8(8),pp.13081339,2011.

[5] C.Yu, W.Fung, H.Lin, Q.Zhang, J.Sanderson. Predictors of left ventricular reverse remodeling after cardiac resynchronization therapy for heart failure secondary to idiopathic dilated or 
ischemic cardiomyopathy. Am J Cardiol.vol. 91(6),pp.684-688,2003.

[6] G.Bleeker, M.Schalij, S.Molhoek, H.Verwey, E.Holman. Relationship between QRS duration and left ventricular dyssynchrony in patients with end-stage heart failure. J Cardiovasc Electrophysiol.vol.15(5),pp. 544-549, 2004.

[7] Javier García-Seara. Influence of the Preimplantation QRS Axis on Responses to Cardiac Resynchronization Therapy .vol.8,pp.1245-1252,2018.

[8] C.Ypenburg, RJ.van Bommel, CJW.Borleffs, GB.Bleeker, E.Boersma. Long-term prognosis after cardiac resynchronization therapy is related to the extent of left ventricular reverse remodeling at midterm follow-up. J Am Coll Cardiol.vol. 53(6),pp. 483-490, 2009.

[9] CA.Henrikson, DD.Spragg, A.Cheng, M.Capps, K.Devaughn. Evidence for electrical remodeling of the native conduction system with cardiac resynchronization therapy. Pacing Clin Electrophysiol.vol. 30(5),pp. 591-595, 2007.

[10] CM.Yu, DL.Hayes. Cardiac Resynchronization Therapy. Eur Heart J.vol. 34(19),pp.1396-1403,2013.

[11] M.St John Sutton, S.Ghio, T.Plappert, L.Tavazzi, L.Scelsi. Resynchronization reverses Remodeling in Systolic left ventricular dysfunction (REVERSE) Study Group. Cardiac resynchronization induces major structural and functional reverse remodeling in patients with New York Heart Association class I/II heart failure. Circulation.vol. 120(19),pp. 1858-1865. ,2009.

[12] FA.Sebag, RP.Martins, P.Defaye, F.HiddenLucet, P.Mabo. Reverse Electrical Remodeling by Cardiac Resynchronization Therapy: Prevalence and Clinical Impact. J Cardiovasc Electrophysiol.vol. 23(11),pp. 1219-1227,2012.

[13] K.Mischke, C.Knackstedt, S.FacheK, Reith, O.Rana. Electrical remodelling in cardiac resynchronization therapy: Decrease in intrinsic QRS duration. Acta Cardiol.vol. 66(2),pp. 175$180,2011$.

[14] J.Dizon, E.Horn, J.Neglia, N.Medina, H.Garan. Loss of left bundle branch block following biventricular pacing therapy for heart failure: evidence for electrical remodelling? J Interv Card Electrophysiol.vol. 10(1),pp. 47-50, 2004.

[15] M.Stockburger, A.Nitardy, S.FatehMoghadam, A.Krebs, O.Celebi, Electrical remodeling and cardiac dimensions in patients treated by cardiac resynchronization and heart failure controls. Pacing Clin Electrophysiol.vol. 31(1),pp. 70-77,2008.

[16] S.Baldasseroni, A.Gentile, M.Gorini, N.Marchionni, M.Marini. Intraventricular conduction defects in patients with congestive heart failure: left but not right bundle branch block is a independent predictor of prognosis. A report from the Italian Network on Congestive Heart Failure (IN-CHF database). Ital Heart J. vol. 4(9),pp.607-613,2003.

[17] LG.Tereshchenko, CA.Henrikson, P.Stempniewicz, L.Han, RD.Berger. Antiarrhythmic effect of reverse electrical remodeling associated with cardiac resynchronization therapy. Pacing Clin Electrophysiol.vol. 34(3),pp. 357-364,2011.

[18] M.Malik, VN.Batchvarov. Measurement, interpretation and clinical potential of QT dispersion. J Am Coll Cardiol.vol. 36(6),pp. 1749-1766,2000.

[19] GX.Yan, SJ.Rials, Y.Wu, T.Liu, X.Xu. Ventricular hypertrophy amplifies transmural repolarization dispersion and induces early after depolarization. Am J Physiol Heart Circ Physiol.vol. 281(5),pp. H1968-H1975, 2001.

[20] Antzelevitch C, Fish J (2001) Electrical heterogeneity within the ventricular wall. Basic Res Cardiol 96: 517-527.

[21] R.Panikkath, K.Reinier, A.Uy-Evanado, C.Teodorescu, J.Hattenhauer. Prolonged Tpeak-to-tend interval on the resting ECG is associated with increased risk of sudden cardiac death. Circ Arrhythm Electrophysiol.vol. 4(4),pp. 441-447,2011.

[22] C.Haarmark, C.Graff, MP.Andersen, T.Hardahl, JJ.Struijk. Reference values of electrocardiogram repolarization variables in a healthy population. J Electrocardiol.vol. 43(1),p. 31-39,2010.

[23] K.Porthan, M.Viitasalo, L.Toivonen, AS.Havulinna, A Jula. Predictive value of electrocardiographic T-wave morphology parameters and $\mathrm{T}$-wave peak to $\mathrm{T}$-wave end interval for sudden cardiac death in the general population. Circ Arrhythm Electrophysiol.vol. 6,pp. 690-696,2013.

[24] C.Ypenburg, RJ. van Bommel, CJW. Borleffs, GB. Bleeker, E.Boersma. Long-term prognosis after cardiac resynchronization therapy is related to the extent of left ventricular reverse remodeling at midterm follow-up. J Am Coll Cardiol.vol. 53(6),pp. 483-490,2009.

[25] K.Chakir, SK.Daya, RS.Tunin, RH.Helm, MJ.Byrne. Reversal of global apoptosis and regional stress kinase activation by cardiac resynchronization. Circulation.vol. 117(11),pp. 1369-1377. ,2008.

[26] J.Benjamin. Sieniewicz, Justin Gould, S.Bradley Porter, Baldeep Sidhu . Understanding non-response to cardiac resynchronisation therapy: common problems and potential solutions. Heart Failure Reviews volume.vol. 24,pp. 41-5,2019.

[27] Anaïs Gauthey ,Erik Willemen . Impact of paced left ventricular dyssynchrony on left 
ventricular reverse remodeling after cardiac resynchronization therapy .journal of cardiovascular electrophysiology .vol. 8,pp.1540-8167, 2020.

[28] Dominique Auger . prevalence and characteristics of patients with clinical improvement but not significant left ventricular reverse remodeling after cardiac resynchronization therapy. American Heart Journal.vol. 160(4),pp.737-43,2010.

[29] J.van 't T.P. Sant, M.M. Mast, I.A.Bos, W.M.ter Horst. van Everdingen. Neth Heart J.vol. 24(1),pp. 47$55,2016$.

[30] Hee-Jin Kwon, Kyoung-Min Park. Electrical ReverseRemodeling of the Native Cardiac Conduction System after Cardiac Resynchronization TherapyJClin Med.vol.9(7),pp.2152,2020. 